UDK 528.225

\title{
FIELD TESTS OF ASTROMETRIC SUBSYSTEM
}

\section{Ansis ZARIN̦Ś ${ }^{1}$, Diāna HARITONOVA ${ }^{2}$, Augusts RUBANS ${ }^{3}$, Gunārs SILABRIEDIS ${ }^{4}$, Jānis ZVIRGZDS ${ }^{5}$}

Institute of Geodesy and Geoinformatics, University of Latvia,

19 Raina Blvd., LV 1586 Riga, Latvia

E-mails: ${ }^{1}$ ansiszx@inbox.lv (corresponding author); ${ }^{2}$ diana.haritonova@inbox.lv;

3augusts.rubans@lu.lv; ${ }^{4}$ gunars.silabriedis@lu.lv; ${ }^{5}$ janis.zvirgzds@lgia.gov.lv

Received 12 November 2015; accepted 02 February 2016

\begin{abstract}
The paper presents results of astrometric subsystem's functionality tests for space object laser ranging and astrometric position determination device, which is under construction in the Institute of Geodesy and Geoinformatics (GGI) of the University of Latvia. Properties of hardware performance for astrometric image acquisition were evaluated and optimal parameters have been selected. Software for image processing and calculation of instrument orientation was tested and adjustments have been made for real-time operation support. Object tracking properties were evaluated, and mount error model parameters were calculated, using test measurements.
\end{abstract}

Keywords: geodetic astronomy, satellite geodesy, satellite laser ranging, image processing.

\section{Introduction}

Astrometric position determination is part of a number of applications, which use star images for instrument orientation or object coordinate calculation. In order to fully employ benefits, offered by modern imaging technologies and reference star catalogues, automatic realtime processing of astrometric data is of considerable importance.

Satellite laser ranging is a particularly demanding application, which requires high and technically exacting accuracy for instrument positioning. Real-time astrometric position availability can be quite useful for positioning system testing and calibration. It would also enable fast determination of instrument or object position for other purposes, in particular, for digital zenith camera’s support (Abele et al. 2012; Zariňš et al. 2014).

Astrometric instruments, employing such technology, presently are being designed in GGI. This paper reflects results of functional tests, as well as adjustment of control software and hardware components for the project of multi-purpose near-Earth object tracking device.

\section{Design of device}

The device is intended for laser ranging and astrometric coordinate determination of near-Earth objects, and is under construction since 2014 using ESF research grant funding. It is being designed as a simple and cheap alternative to custom-made systems, which tend to be quite expensive. Therefore, the design includes many off-the-shelf commercial components.

The design includes twin 16" $(42 \mathrm{~cm})$ catadioptric optical tube assemblies (OTA) on stepper motor driven Alt-Alt mount (Figs 1,2). Although usage of both OTA can be configured in various ways, the basic configuration will include reflected pulse detector fitted to one (a collimator for transmitted pulse mounted

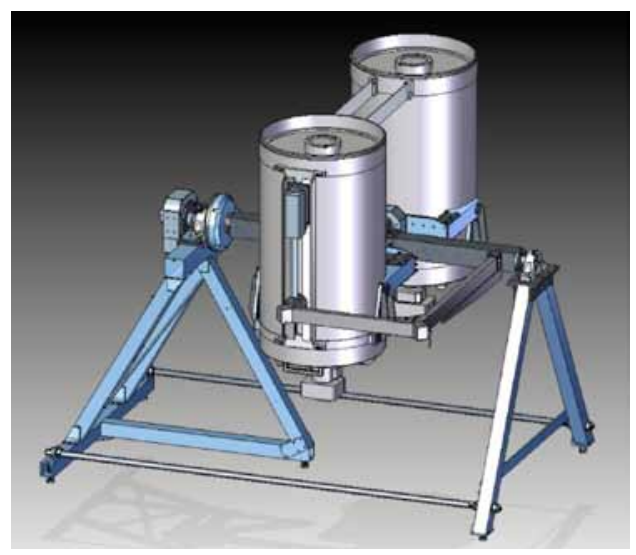

Fig. 1. Design of tracking device. Coudé path for transmitted laser beam enters along horizontal primary axis. Transmitting collimator is attached to the side of receiving OTA 


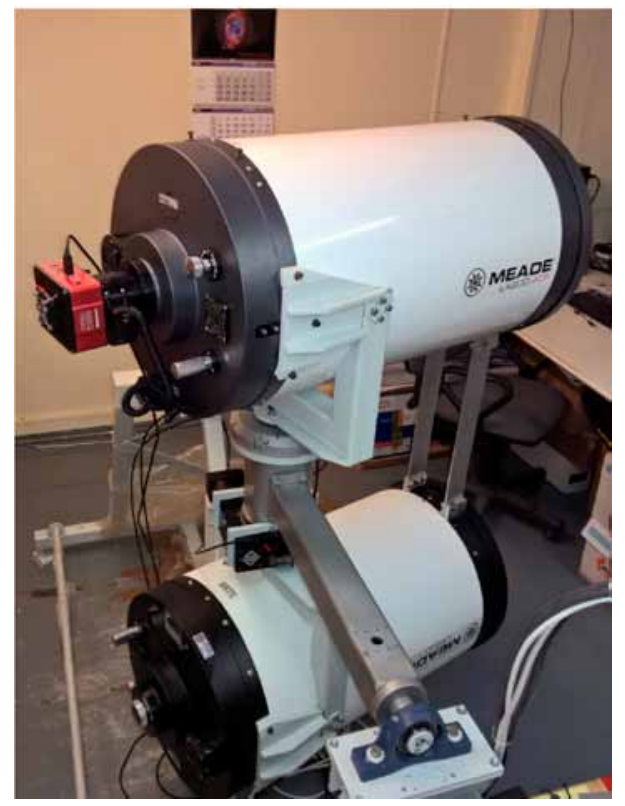

Fig. 2. Assembly of tracking device.

on side of OTA), and the other OTA will be equipped with imaging device for astrometric purposes. Besides general astrometric imaging, independent of the SLR functionality, astrometric subsystem's tasks will include visual guiding, determination of current orientation of system for real-time pointing corrections, as well as data acquisition for mount error model calculations.

In late 2015 the device was brought out for field tests of astrometric subsystem. The main objectives of the field tests are evaluation of performance capabilities and determination of parameters for optimal imaging and data processing.

\section{Star image acquisition tests}

Presently device is equipped with a 8 Mpix CCD camera with following parameters:

- pixel size - $5.4 \mu$

- CCD dimensions - $18 \times 13.5 \mathrm{~mm}$;

- field of view $-0.27 \times 0.2 \mathrm{dg}$;

- image scale - 0.29"/pixel.

Besides, full resolution, also $2 \times 2,3 \times 3$ and $9 \times 9$ pixel binning modes are available.

Image reading and processing are much faster in binning mode, there is also some sensitivity gain. Camera has no direct hardware support for accurate image timing. If accuracy in millisecond level is not essential (e.g., in the case of long exposures), computer time of image acquisition request can be used, applying appropriate corrections for exposure delay and computer clock shift. Accuracy up to about 10 milliseconds can be ensured in this way, which is quite sufficient for most astrometric tasks, so presently we get along with it. More accurate timing can be achieved registering mechanical shutter "open/close" signal events using some timing reference (for example, GPS receiver event timing mechanism).

As our site's location doesn't have fine astroclimate (close to the sea, high humidity, haze, background lights due to proximity of a big city), obtained image acquisition results probably are somewhat biased. Better results might be expected in improved conditions. In particular, although theoretically OTA should provide near-diffraction resolution of better than 1", the smallest practically achieved size of star image was about 3". In these circumstances it has been found that usage of full CCD resolution is not the best choice, and $2 \times 2$ pixel binning mode gives very similar accuracy, but is much faster, because of $4 \times$ smaller data amount to be read from CCD, stored in a file and processed, and gives some advantage in registered star magnitudes besides.

Imaging sensitivity, achieved during tests, is summarized in Figure 3. The figure represents dependency of maximum star magnitude and number of stars in frame on exposure duration. As CCD outcome is strongly dependent on star's color, and many weaker stars have no visual or photographic magnitude estimates (the magnitude, representing the color, which was closest to visual for the star has been used in these cases), maximum magnitude only approximately represents sensitivity. Minimum exposure for the camera is 0.12 seconds. For static imaging (mount isn't moving) of areas near celestial equator exposures above 0.2 seconds perceptibly elongate images due to Earth rotation, undoing longer exposure gains - number of star images doesn't increase any more. Images of

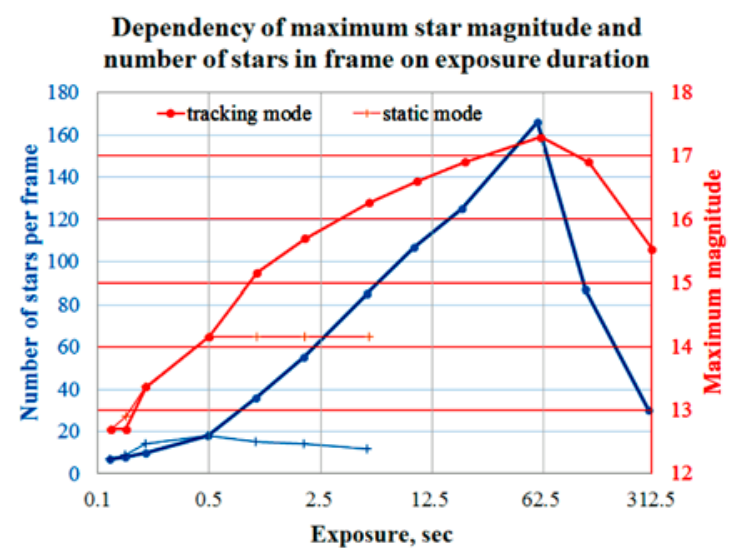

Fig. 3. Number of star images on frame and biggest magnitude for both static and tracking imaging modes

Note: a randomly selected sky area, close to zenith 
mid-latitude areas are significantly elongated for exposures above 0.3 seconds, and in the case of exposures above 0.5 seconds elongation is too big everywhere except polar areas. Therefore, exposure duration about $0.2-0.3$ seconds seems to be optimal for static imaging.

Tracking accuracy of our device is limited by minimum displacement, corresponding to step of a stepper motor, which in our case is about 0.5 ". On top of that, control software is performing adjustments of tracking only when coordinate discrepancy increases above a configurable threshold. At angular encoder resolution of 0.37 ", it was established that there is no benefit to set this threshold below 1". So, tracking accuracy cannot be better than 1 " -1.5 ", it occasionally experiences external perturbations, causing bigger bounces. Such tracking a bit, but not significantly, enlarges star images in comparison to short-exposure static mode.

Image acquisition tests in tracking mode (Fig. 3) indicate similar results to static imaging for short exposures, and steady increase in number of stars and maximum magnitude if exposure is increased up to about one minute. Further increase of exposure produces orioles around brightest stars, masking faint stars, which are present there. Probably, better astroclimate and instrument location would eliminate much of this effect. Exposures over about 5 minutes show effect of field rotation, elongating star images in the periphery of frame. Without de-rotation equipment, a few minutes (or up to 10-20 minutes in the center of frame) seem to be the technical limit for star field exposures by the device.

Maximum star magnitude in the results mentioned above refers to star images with certainty recognized automatically. In our circumstances the best of it was a bit above $17^{\mathrm{m}}$ for one minute exposure, probably, it would be somewhat higher in good astroclimate. Closer inspection of images typically allows to recognize signs of objects, which are fainter for about one magnitude.

In order to successfully perform automatic star identification (software package described in (Zariņš et al. 2014) was used), at least 5-6 star images should be present; bigger number of stars is desirable to increase astrometric reduction accuracy. For used hardware layout, frames obtained in a random sky area in static mode have provided necessary minimum number of images in about $80 \%$ of cases. There could be two options, how it is possible to increase success rate - either to take additional frames in adjacent areas, or to use tracking mode and longer exposure. Both show similar results and require some moving of device. The choice probably should depend on priorities and limitations of the task.

\section{Mount error model}

One of principal design goals for the device was laser ranging capability. To perform laser ranging in automatic ("blind") mode, pointing accuracy must be better than transmitted beam divergence (the other limiting component - field of view of reflected pulse detector; usually is bigger than divergence). As subarcminute beam divergence is a common choice, and it can be made a fraction of arc minute for ranging of far objects. Consequently, pointing accuracy ideally should be within a few arc second range. It is difficult to ensure such accuracy by purely mechanical means; a very massive, accurately manufactured and expensive support structure would be needed. Instead, a mount error model, implemented in control software, was used to introduce necessary pointing corrections.

A problem is to find a simple mathematical model, effectively describing physical deformations and misalignments of mount. It is almost impossible to construct a reliable theoretical deformation model, including contributions of all physical components and their interactions. Therefore, approximating models, based on position measurement data, are being used instead.

Simplest approaches are models using power series or trigonometric series, or their combination. Also variations of cell- or zone- based models have been used (Zhaborovsky et al. 2012; Medvedsky, Pap 2008; Medvedsky, Suberlak 2002). Trigonometric models include some first members of Fourier series for both coordinate angles and some cross-products; additionally components of an analytical deformation model can be included (for example, as proposed in ILRS (2015)). Such models should reliably describe simple mount misalignments and rotation deformations. On the other hand, they have proved to be not so suitable in case when character of deformations is complex. Power series' models (kind of Turner transformation (Podobed, Nesterov 1975)) can be more reliable if complex deformations are dominating.

Capabilities of described device allow to perform acquisition of measurement data for mount error model calculation in an automated process - to control system a scenario file is provided, containing desired measurement positions. The set of positions is traversed at a rate of about one position per 10 seconds, taking one or several images in each position. Data 
processing can be done either immediately after each frame, or when all frames are obtained. Processing results contain both position coordinates in instrument system (axes rotation angles $\mathrm{C} 1$ and $\mathrm{C} 2$ as acquired from angular encoders) and measured positions in topocentric system. Measured topocentric coordinates are transformed to instrument coordinates $\mathrm{C} 1$, $\mathrm{C} 2$, using the same procedure as for pointing targets. Mount error model then approximates difference of measured and instrument coordinates.

Testing a number of combinations of model member functions, it has been found that more reliable results with least number of model parameters offer a model, consisting of power series. Inclusion of some trigonometric member functions (in particular, sinC $1 \cdot \operatorname{tgC} 2, \cos C 1 \cdot \operatorname{tgC} 2)$ gave almost the same accuracy, but at bigger number of parameters. For the coordinate range, available for position measurements at the test site, no advantage has been found for trigonometric member functions. However, bigger coordinate range might present different situation. Presently adopted structure of the model and the example set of calculated parameters are shown in Table 1.

Measurement data for this model represented more than 100 positions mostly above $\approx 50$ dg elevation (lower elevations were not available because of observation site limitations; two frames were taken at all positions). An example of distribution of measurement positions and corresponding approximation residuals are shown in Figure 4. A number of similar observation sets was obtained. Apart from reflecting mechanical adjustments of the mount made between sessions, no significant variations of resulting mount error models were found.

Table 1. Member functions of mount error model and values for the coefficients (in arcseconds/radian)

\begin{tabular}{|c|c|c|}
\hline Function & $\mathrm{dC} 1$ & $\mathrm{dC} 2$ \\
\hline offset & 267 & -592 \\
\hline $\mathrm{C} 2$ & -34 & -419 \\
\hline $\mathrm{C} 2^{2}$ & -92 & -250 \\
\hline $\mathrm{C} 2^{3}$ & -12 & 471 \\
\hline $\mathrm{C} 1$ & -285 & 59 \\
\hline $\mathrm{C} 1^{2}$ & -101 & 620 \\
\hline $\mathrm{C} 1^{3}$ & 160 & -332 \\
\hline $\mathrm{C} 1 \cdot \mathrm{C} 2$ & -1252 & 104 \\
\hline $\mathrm{C} 1 \cdot \mathrm{C} 2^{2}$ & 19 & -113 \\
\hline $\mathrm{C} 1^{2} \cdot \mathrm{C} 2$ & -402 & 744 \\
\hline
\end{tabular}

3-D representation of mount error model behavior (for each axis separately) is shown in Figure 5a, b. Offset values represent encoder zero-point marker position relative to instrument coordinate system zeropoints; for better representation of variability they are removed from graphs. Asymmetric character of model graphs probably is result of axes non-orthogonality with value of 2-4 arc minutes. Considerable part of mount errors might be the result of deformations, caused by unbalancing of axes. Further experiments with different balancing properties will be made to find out its effects. Correction members, proportional to the other axis value ( $\mathrm{dC} 1 \sim \mathrm{C} 2, \mathrm{dC} 2 \sim \mathrm{C} 1$ ), depend, besides other effects, on azimuth offset of primary axis. Minimization of these member coefficients was used to improve primary axis's azimuth value, used in coordinate transformation from horizontal to instrument system.

Rms value of approximation residuals typically was a little below 10". Similar results have been obtained using data from several sets of observations; difference between respective model values was within $10-20$ ". Model is definitely a simplification of the real situation, therefore a part of approximation residual value reflects inability of model to adhere to detailed structure of real mount errors, which represent combined input from a number of sources: encoder irregularity (up to 2-3"), positioning system residuals ( 1"), astrometric position errors (estimated to be better than 1 "), irregularities of axes shape and bearings (probably the dominating source). Possibly effects of some nonelastic or temperature-inflicted deformations were also

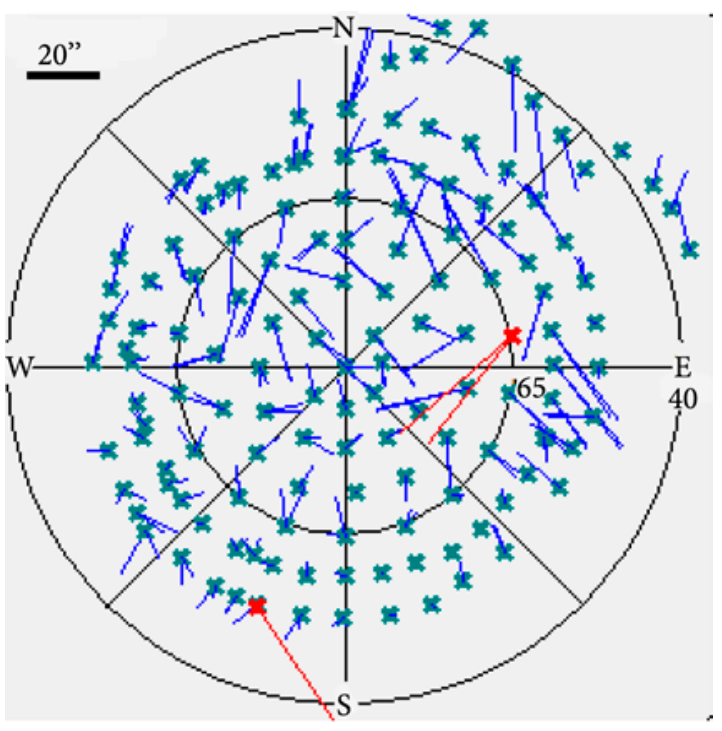

Fig. 4. Measurement point positions of the mount error model and approximation residuals Note: rejected measurements are marked red. 
a

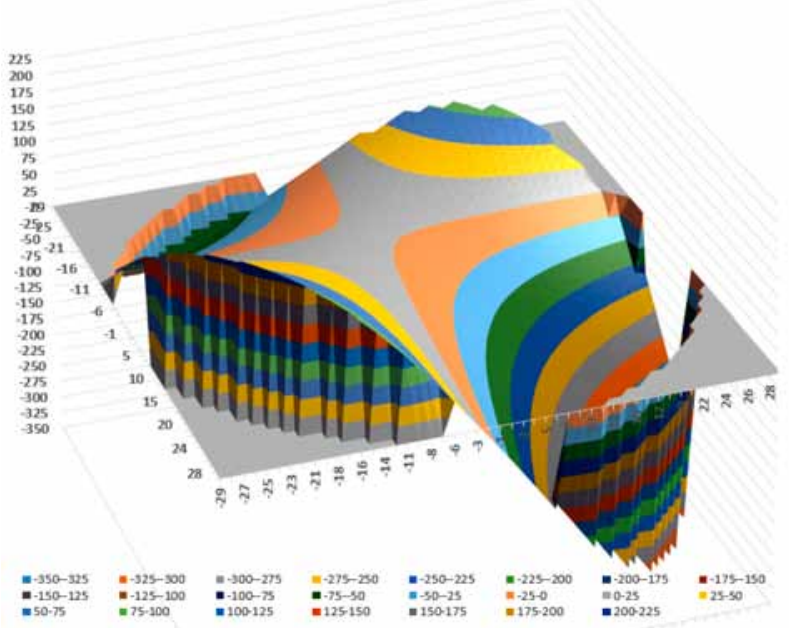

$\mathrm{b}$

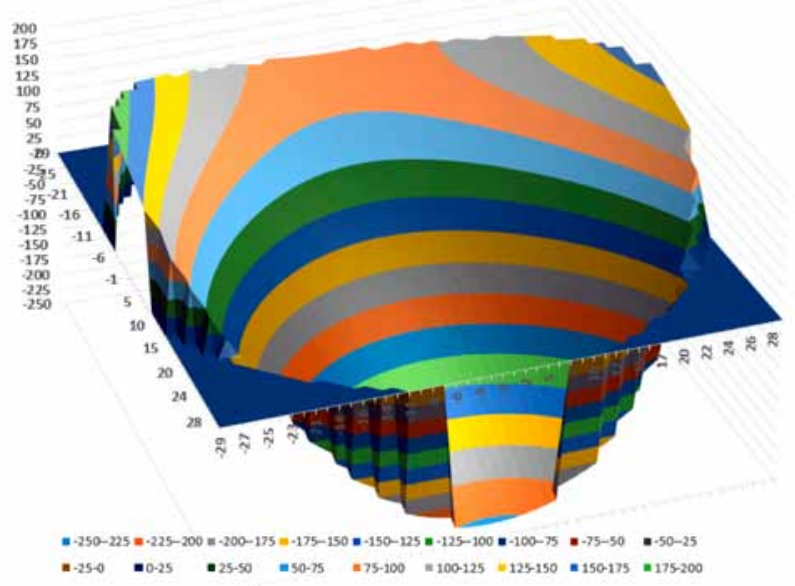

Fig. 5. Mount error model: a - corrections of primary $(\mathrm{C} 1)$ axis rotation as functions of position; $\mathrm{b}$ - corrections of secondary $(\mathrm{C} 2)$ axis rotation as functions of position.

present. It can be expected that regular executing of mount error model measurements hereafter will provide more insight into sources and character of mount positioning irregularities.

Application of calculated mount error model has ensured resulting positioning accuracy generally within $10-20$ ", that should already be adequate for "blind" loworbit satellite ranging. At that accuracy level some searching may be necessary for high-orbit satellites, if very small transmitted beam divergence is used. Hopefully, further adjustment of mount mechanics and improvement of mount error model structure will eventually reduce positioning accuracy to $5-10$ " or less.

\section{Conclusions}

Near-Earth object tracking device's astrometric subsystem's field tests have shown imaging and positioning performance close to what was expected for the design. Mount error model parameters were calculated; resulting positioning accuracy is already adequate for SLR purposes, with prospects of improvement by adjustment of mount mechanical properties and error model structure. Magnitude limit for astrometric position determination was found to be about $17^{\mathrm{m}}$, with possibility of some increase in good imaging conditions. Real imaging and positioning environment induced a number of adjustments in control software. Some mechanical problems have been found and will be treated before final installation of the instrument.

\section{Acknowledgment}

The research was funded by ESF, project No 013/0066/1DP/1.1.1.2.0/13/APIA/ VIAA/059.

\section{References}

Abele, M.; Balodis, J.; Janpaule, I.; Lasmane, I.; Rubans, A.; Zarinšs, A. 2012. Digital zenith camera for vertical deflection determination, Geodesy and Cartography 38(4): 123-129. http://dx.doi.org/10.3846/20296991.2012.755324

ILRS / Technology / Modeling [online], [cited November 2015]. Available from Internet: http://ilrs.gsfc.nasa.gov/technology/ modeling/index.html

Medvedsky, M.; Pap, V. 2008. The new pointing model of telescope based on tracking data, in Proceedings of the 16th International Workshop on Laser Ranging, 12-17 October 2008, Poznan, Poland, 483-488.

Medvedsky, M. M.; Suberlak, V. R. 2002. Mount errors model for the Kyiv SLR station, Artificial Satellites 37: 3-16.

Podobed, V. V.; Nesterov, V. V. 1975. General astrometry. Moscow: Nauka (in Russian).

Zariņš, A.; Janpaule, I.; Kaminskis, J. 2014. On reference star recognition and identification, Geodesy and Cartography 40(4): 143-147.

http://dx.doi.org/10.3846/20296991.2014.987456

Zhaborovsky, V. P.; Pap, V. O.; Medvedsky, M. M.; Choliy, V. Ya. 2012. Error budget model for SLR station 1824 KyivGolosieve telescope, Bulletin of Ukrainian Earth Orientation Parameters Laboratory 7: 27-29.

Ansis ZARIN̦Š. Senior researcher at the Institute of Geodesy and Geoinformatics, University of Latvia, 19 Raina Blvd., LV 1586 Riga, Latvia (Phone: +371 67034435), e-mail: ansiszx@inbox.lv. (Dr.phys. 1988). Research interests: control and data processing systems for satellite observation and astrometric instruments.

Diāna HARITONOVA. Researcher at the Institute of Geodesy and Geoinformatics, University of Latvia, 19 Raina Blvd., LV 1586 Riga, Latvia (Phone: +371 67034435), e-mail: diana. haritonova@inbox.lv. (Mg.sc.ing. 2012). PhD student at Riga Technical University, the Faculty of Civil Engineering. Research interests: analysis of GNSS station time series, Earth's surface displacements. 
Augusts RUBANS. Researcher at the Institute of Geodesy and Geoinformatics, University of Latvia, 19 Raina Blvd., LV 1586 Riga, Latvia (Phone: +371 67034436), e-mail: augusts@lu.lv. (Mg.sc.phys. 1993). Research interests: design of astrometric equipment, SLR, GNSS.

Gunārs SILABRIEDIS. Director and senior researcher at the Institute of Geodesy and Geoinformatics, University of Latvia, 19 Raina Blvd., LV 1586 Riga, Latvia (Phone: +371 67034436), e-mail: gunars.silabriedis@lu.lv. (Dr.sc.ing. 2013). Research interests: GNSS data, height systems.

Jānis ZVIRGZDS. Senior researcher at the Institute of Geodesy and Geoinformatics, University of Latvia, 19 Raina Blvd., Riga, LV 1586, Latvia (Phone: +371 29259135), e-mail: janis. zvirgzds@lgia.gov.lv. (Dr.sc.ing. 2012). Head of GPS Network laboratory of the Latvian Geospatial Information Agency. Research interests: GNSS data processing and analysis. 